\title{
Editorial
}

Johannes J.M.L. Hoffmann

\section{Observing an analyzer's operational life cycle: a useful management tool for clinical laboratories}

https://doi.org/10.1515/cclm-2018-0725

In the process of sourcing new equipment for the clinical laboratory, analytical performance of the analyzer, ease of operation and cost are the dominant factors on which the final buying decision is based. Vary rarely, if ever, considerations on expected lifespan of an analyzer do play a role, and if these factors are being considered, they are usually based on the manufacturer's reputation or on hearsay from colleagues using the same analyzer or analyzer brand, rather than on solid evidence. In addition, after installation of a new analyzer in the laboratory, there is generally not much attention for the failure rate (reliability) and maintenance need other than log books and empirical findings of the laboratory staff. The current issue of the journal contains an excellent paper by Verma and colleagues, who present the Weibull statistics for accurately quantifying the failure rate and predicting the life cycle of hematology analyzers [1]. This statistical method is widely used for reliability analysis and fatigue testing in engineering, but until now, it has not been applied to clinical laboratory equipment, and thus, the authors deserve our appreciation for bringing this subject into the field of laboratory medicine. The Weibull method transforms instrument failures observed during a certain period into a probability function that can be plotted for easy visual interpretation and that allows for an objective prediction of life cycle and failure rate. Although the Verma paper is on hematology analyzers, the principle can be equally well be applied to other analyzer types or other equipment that is critical for a clinical laboratory's function. When and why can this be useful?

Following analyzer implementation in the laboratory. Usually, a new analyzer has an initial failure rate that is higher than later on in its operational life. This is mainly due to the laboratory staff not yet having reached the plateau phase of learning how to handle and maintain the analyzer. However, the initial failure rate may also be higher as a consequence of unforeseen issues in the design of the analyzer, particularly when a new analyzer was recently launched to the market. During the design of a new analyzer, instrument manufacturers use failure rates of the key mechanical and electronic components for calculating the expected failure rate, generally expressed as mean time between failures (MTBF) of the entire system. If necessary, components that fail too often are replaced to meet the commercial MTBF requirements of the new analyzer. In the final phase of development, just before commercial launch, manufacturers verify if the actual MTBF is in accordance with the estimated MTBF. However, these reliability data are usually collected in strictly controlled conditions that do not accurately reflect routine practice in clinical laboratories and therefore, the failure rate may be underestimated. Moreover, such reliability data are not generally shared with (potential) buyers. Of course, the manufacturer's recommendations for preventive maintenance frequency have a certain relationship with known failure data, but the exact connection remains obscure for the end user. Therefore, laboratories have to build up their own experience with the analyzer and its failure rate. This is where the Weibull method may be useful, as is demonstrated in the paper by Verma et al. [1]. If the failure rate of a new analyzer would be higher than anticipated, the laboratory would certainly have a case for discussing the reliability with the manufacturer, supported by quantitative data. Eventually, this may help improving the operational performance of the analyzer by remedying the childhood diseases and accelerating the learning curve of analyzer handling and maintenance for the laboratory staff.

Monitoring the analyzer's operational life. Measuring failure rates of individual analyzers does not help predict when failure occurs and neither does it prevent operational downtime. However, on the longer term, it may yield valuable information on analyzer condition from a laboratory management perspective. This is particularly useful if the laboratory operates multiple identical instruments in parallel, or alternatively, if a laboratory would compare its Weibull data with other laboratories, for example in a regional or national analyzer user group or as sort of external reliability control program for the instrument.

Logically, the failure rate of an instrument tends to increase when it is more extensively used and when it becomes older. By comparing historical and actual failure 
rates, the effect of changes in use, of corrective interventions and of modifications in preventive and reactive maintenance can be objectively assessed and one may also calculate whether the maintenance and service costs remain within acceptable limits. According to the 'bathtub' model [1], any instrument will eventually progress from its useful operational life phase to the 'wear out' stage, and this transition can be timely observed by proactive monitoring of operational reliability. If such a transition appears to occur, appropriate measures can be taken, like intensified preventive maintenance or changing the pattern of use, for example by measuring samples from less critical patients. Evidently, these are only temporary measures, until the analyzer has reached the wear-out phase and a more definitive solution needs to be applied.

The wear-out phase. In this phase, the instrument starts to show increasingly more frequent failures that herald the end of its useful operational and economical life. In this phase, Weibull analysis can supply objective data for determining when replacing the instrument is more economical than continued intensive maintenance and service. From a financial management perspective, a business case based on objective Weibull data is likely to be more successful.

Now more and more laboratories use sort of dashboard or cockpit where the entire automated laboratory process is continuously monitored, and it would be advisable to integrate the Weibull statistics in the monitoring software to provide the management with a regular overview of the operational status of the instruments. As soon as an adverse trend in reliability becomes evident, there is probably sufficient time for taking proper action without jeopardizing the operational service level of the instrument.
Over the last few years, the diagnostic industry has been using 'partnership' as a marketing mantra. Obviously, there is nothing against a close partnership between supplier and end user, but in my opinion, true partnership requires a more tantamount input than is currently the case. The reliability subject discussed here is a good example, as the ownership and use of failure rate analysis lie still rather one-sided in the hands of the suppliers. The time has definitely come to start sharing data on operational reliability of instruments between manufacturers and end users. Analyzer life cycle management, based on the Weibull concept of failure analysis as shown by Verma and colleagues, would be a good opportunity for a more balanced partnership and would eventually be in favor of the patients who depend on laboratory medicine.

Author contributions: The author has accepted responsibility for the entire content of this submitted manuscript and approved submission.

Research funding: None declared. Employment or leadership: None declared. Honorarium: None declared.

\section{Reference}

1. Verma A, Narula A, Katyal A, Yadav SK, Anand P, Jahan A, et al. Failure rate prediction of equipment: can Weibull distribution be applied to automated hematology analyzers? Clin Chem Lab Med 2018;56:2067-71.

Johannes J.M.L. Hoffmann, H3L Consult, Nuenen, The Netherlands, E-mail: h3l.consult@gmail.com. http://orcid.org/0000-0003-1009. 4568 